\title{
Addressing Post-COVID Symptoms: A Guide for Primary Care Physicians
}

\author{
Heather Vance, MD, Amelita Maslach, MD, Emily Stoneman, MD, \\ Kathryn Harmes, MD, Andrea Ransom, NP, Katharine Seagly, PhD, and \\ Wendy Furst, $M A$
}

Background: Post-COVID symptoms, defined as symptoms lasting $>4$ weeks postinfection, have been identified not only among those patients who were hospitalized with severe symptoms but also among those who were asymptomatic or with only mild symptoms. Primary care providers (PCPs) will often be the first point of contact for patients experiencing potential complications of post-COVID symptoms. The aim of this article is to present a post-COVID management tool for PCPs to use as a quick reference and guide to the initial workup and management of the most common post-CoVID symptoms.

Methods: Published guidance, recent literature, and expert specialist opinion were used to create the structure outlining the outpatient evaluation and treatment for post-COVID symptoms.

Results: A quick-reference guide for management of post-COVID symptoms was created for PCPs. Educational materials were created for clinicians to share with patients. Our article reviews several common complaints including respiratory, cognitive, and neurological symptoms, chronic fatigue, dysautonomia, and anosmia and presents recommendations for management.

Conclusions: Data on long-term effects of COVID-19 are still emerging, and rapid dissemination of this data to front-line PCPs is crucial. This table was our effort to make the currently available evidence accessible for our PCPs in a simple, easy-to-use format. (J Am Board Fam Med 2020;34:1229-1242.)

Keywords: COVID-19, Long-COVID, Multidisciplinary Communication, Patient Care Team, Primary Health Care

\section{Background}

Over the past year, more than 30 million Americans were diagnosed with COVID-19 caused by SARS$\mathrm{CoV}-2,{ }^{1}$ and there is increasing evidence of the enduring effects of COVID-19. ${ }^{2-7}$ While there is a lack of consensus regarding terminology, ${ }^{8}$ symptoms that are present beyond 3 to 4 weeks have been included in much of the research examining the long-term effects of COVID-19. Symptoms lasting

This article was externally peer reviewed.

Submitted 22 June 2021; revised 8 July 2021; accepted 19 July 2021.

From University Health Service, University of Michigan, Ann Arbor (HV); Primary Care Clinic, University Health Service, University of Michigan, Ann Arbor (AM); Department of Internal Medicine, Division of Infectious Diseases, University of Michigan Medical School, Ann Arbor (ES); Department of Family Medicine, University of Michigan Medical School, Ann Arbor (KH); University Health Service, University of Michigan (AR); Division of Rehabilitation Psychology and Neuropsychology, Department of Physical Medicine and Rehabilitation, University of Michigan Medical School, Ann Arbor (KS); Department of Family Medicine, University of Michigan Medical School, Ann Arbor (WF). Funding: None.

Conflict of interest: None. up to 4 weeks after diagnosis are referred to as acute COVID-19, and those symptoms that persist or emerge related to COVID-19 past 12 weeks of infection are labeled long COVID, postacute sequelae of SARS-CoV-2 infection, or chronic COVID-19. This article will use the term post-COVID to include symptoms lasting for more than 4 weeks.

Post-COVID syndrome has been identified not only among those patients who were hospitalized with severe symptoms but also among those who were asymptomatic or with only mild symptoms. ${ }^{7}$ One study with a sample size of 1407 found nearly $30 \%$ of their nonhospitalized COVID-19 participants were still reporting symptoms 2 months after infection. ${ }^{6}$ Further, based on a sample of more than 2000 participants, less than $1 \%$ of both hospitalized and nonhospitalized patients indicated they were symptom-free nearly 80 days after infection. ${ }^{7}$

Corresponding author: Heather Vance, MD, University of Michigan, Ann Arbor, MI (E-mail: hbidgoli@med.umich. edu). 
Among a smaller sample, nearly $70 \%$ of 130 COVID-19 patients reported at least 1 persistent symptom at 2 months after symptom onset. ${ }^{10}$

Post-COVID symptoms may exhibit differently among age and gender cohorts. ${ }^{11}$ Huang et $\mathrm{al}^{6}$ found nearly 3 of every 4 patients who reported lasting symptoms from their sample were 50 to 59 years of age. In contrast, Walsh-Messinger and collegues ${ }^{12}$ found that among 43 college students recovering from COVID-19, half reported persistent symptoms, and all but 1 of these patients were female. Primary reported symptoms include chest pain, dyspnea, anxiety, and nausea. ${ }^{6}$ Goërtz et $\mathrm{al}^{7}$ found fatigue and dyspnea to be the most common within their sample of patients from the Netherlands and Belgium followed by heart palpitations, diarrhea, ageusia, and insomnia. In a follow-up study of 1733 patients in Wuhan, China, $63 \%$ of the patients who identified at least 1 symptom at a 6-month follow-up (76\% of sample) reported fatigue or muscle weakness. ${ }^{6}$ Memory loss and the inability to concentrate, also referred to as "brain fog," have also been associated with post-COVID symptoms. ${ }^{2,12}$ In December 2020, the Office for National Statistics ${ }^{13}$ in the UK found that among 8193 patients, $21 \%$ reported fatigue, cough, and headache as primary symptoms 5 weeks following infection.

Primary care providers (PCPs) will often be the first point of contact for patients experiencing potential complications of post-COVID symptoms. ${ }^{14}$ In addition, as it seems that not all symptoms emerge during the infectious period, PCPs must be prepared to identify, evaluate, and manage these post-COVID complications. ${ }^{15}$ Currently, there is very little evidence-based guidance for PCPs in management of post-COVID symptoms. The aim of this article is to present a post-COVID management tool based both on the emerging but as yet very preliminary evidence base as well as expert consensus, with the understanding that guidelines will evolve over time as additional evidence is brought forth: PCPs will be able to use the included table as a quick reference and guide to the initial workup and management of the most common post-COVID symptoms. This article will also help PCPs facilitate the management of these symptoms and serve as a crucial link to specialty clinics. ${ }^{11}$

\section{Methods}

The University of Michigan's (UM's) Campus Health Response Committee (CHRC) identified a need for practice guidelines pertaining to post-COVID symptoms and invited collaboration from the Michigan Medicine (MM) Hospital System including Primary Care, Occupational Health Service, Infectious Disease, Physical Medicine and Rehabilitation, and University Student Health Service to serve the clinical needs of the UM community. To prepare for this project, team members performed an extensive literature review to further identify the existing information related to post-COVID symptoms. In addition, team members attended a Centers for Disease Control and Prevention (CDC) Clinician Outreach and Communication Activity (COCA) on treating postCOVID symptoms. Following this, members reached out to COCA speakers for additional, extended discussions. Using CDC and National Institutes of Health (NIH) guidance, recent studies that evaluate for postCOVID symptoms, and expert specialist opinion, team members created the structure for a clinician guide outlining the outpatient evaluation and treatment for post-COVID symptoms. Educational materials were created for clinicians to share with patients via the electronic medical record or discuss during their visit. This educational material was subsequently made publicly available through the UM Maize and Blueprint, which is a consolidated resource that includes all COVID-related topics for the UM community. The hope was to make this information as widely available as possible.

While creating this guideline and seeking out MM specialty guidance, it was discovered that MM was concurrently creating a multidisciplinary clinic for post-COVID care. The team was able to collaborate in creating guidance on outpatient evaluation and when specialty care referral may be appropriate. Once the tool was created, it was presented to target MM audiences that comprise most of adult primary care including Internal Medicine, Family Medicine, and University Health Service. The tool and educational materials were also made available to all MM providers through the organization's intranet.

\section{Findings}

Postacute and chronic COVID have been associated with a myriad of symptoms. The PCP should be prepared to assess these symptoms systematically and address accordingly. Many of these symptoms (anxiety, depression, headaches, and so forth) are common complaints in the primary care scope of 


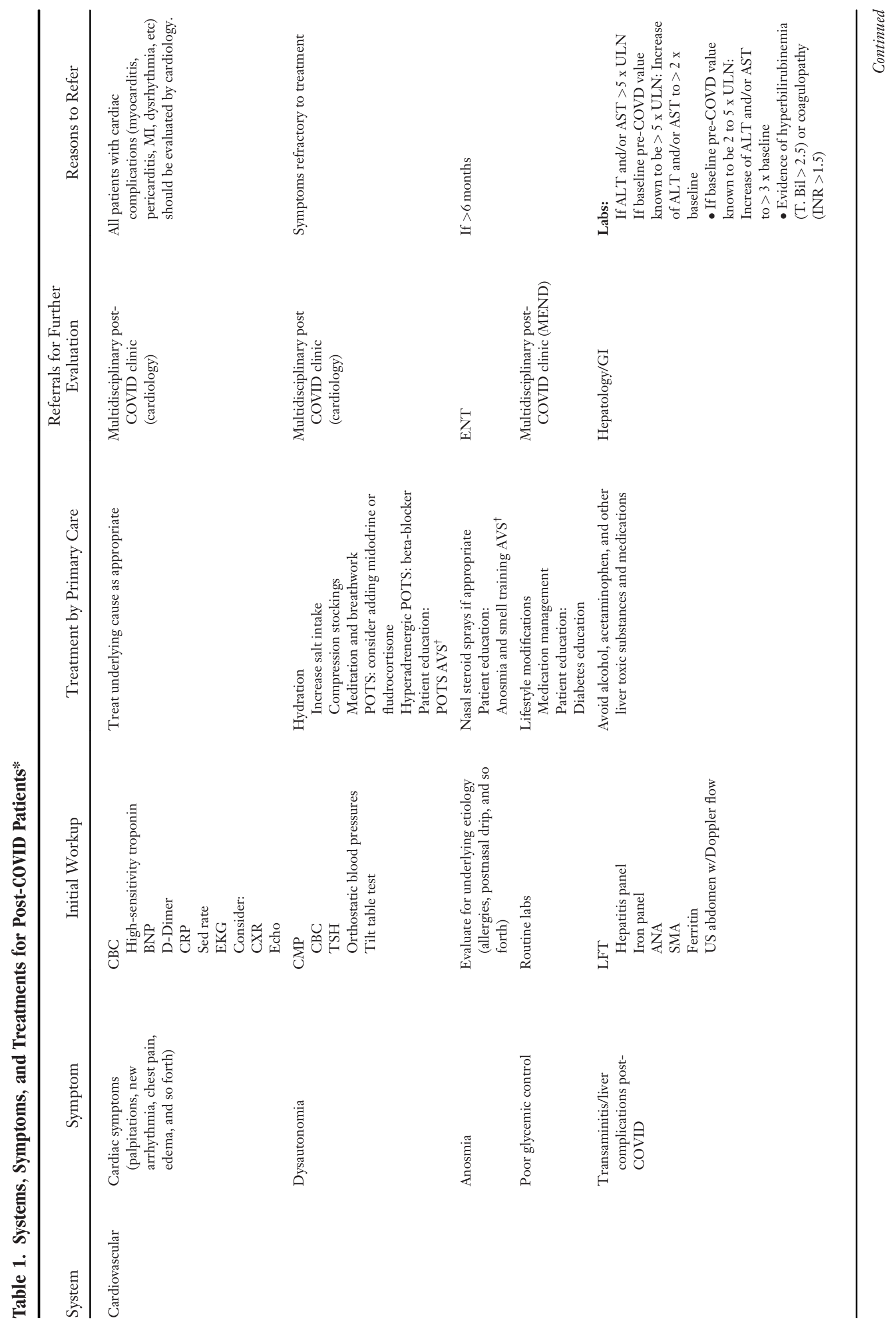




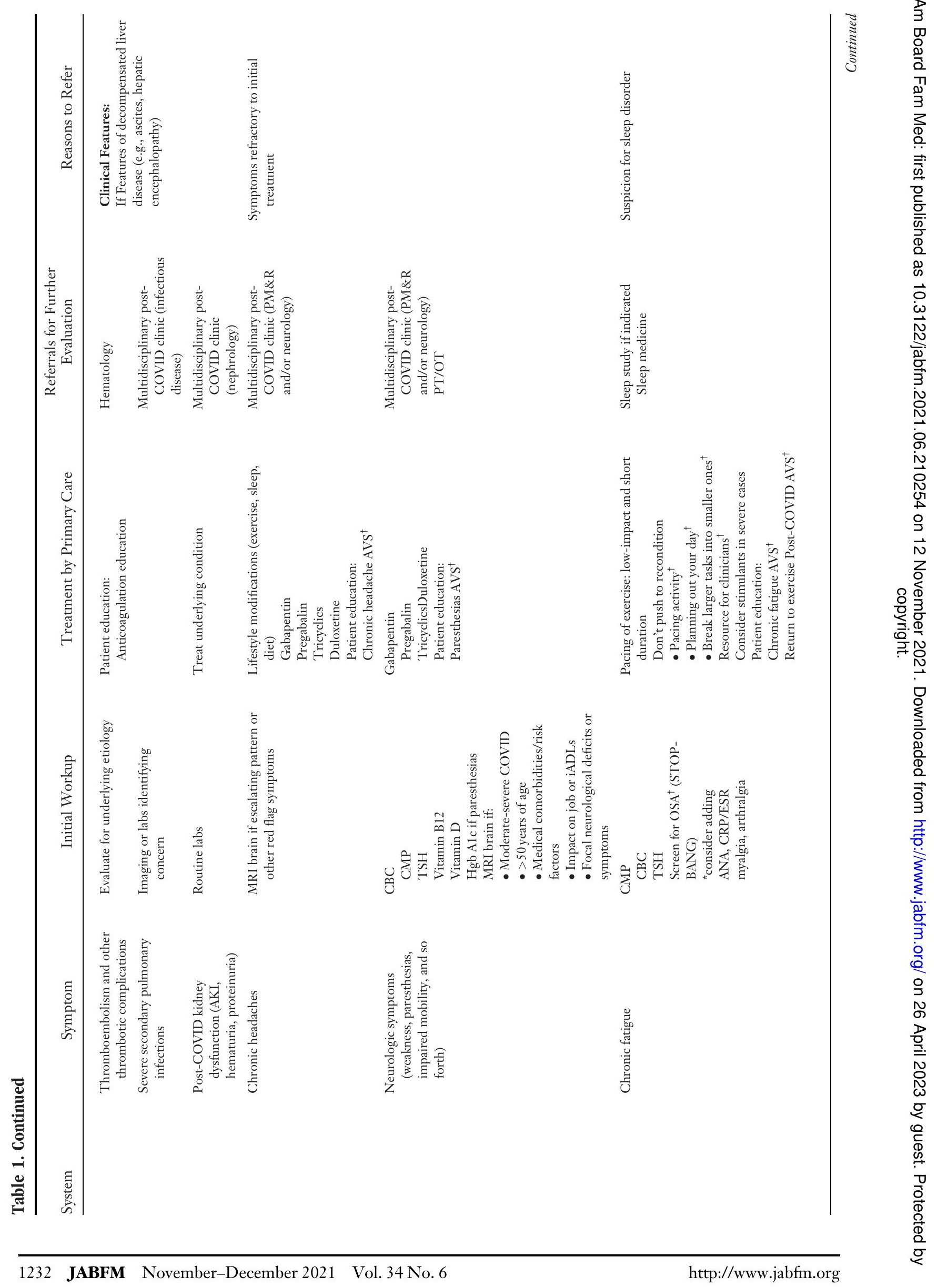




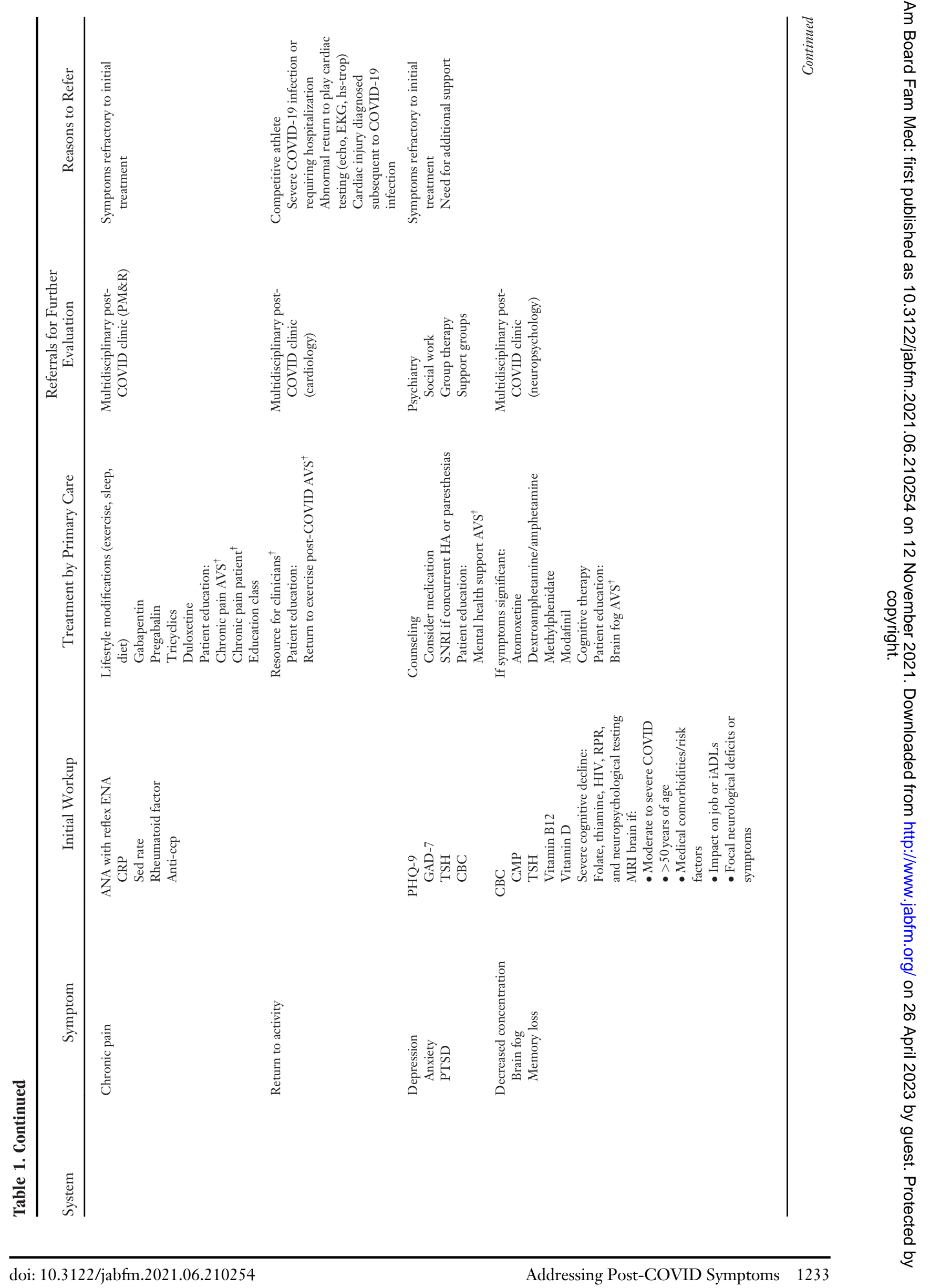




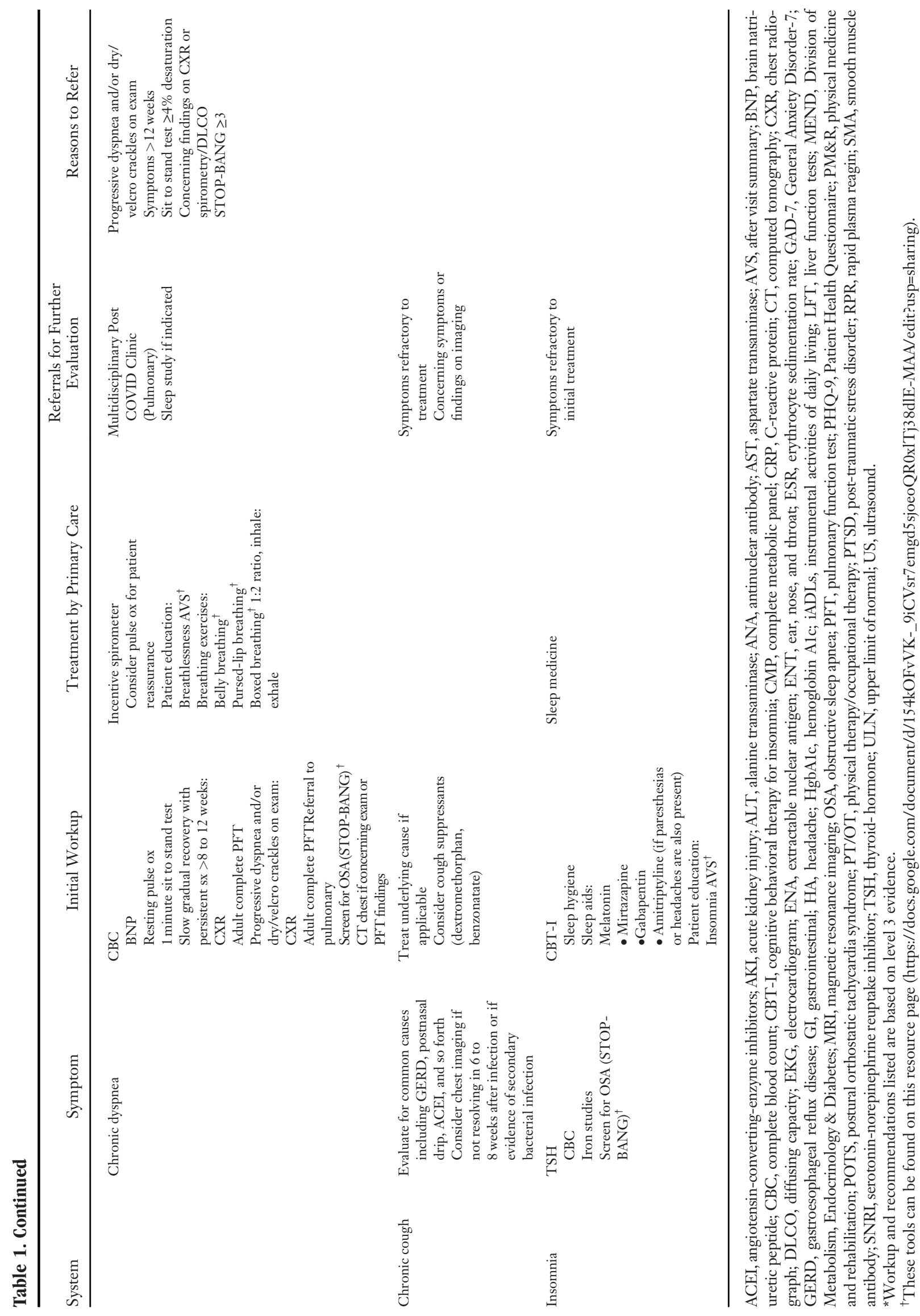


practice and can be addressed by known and accepted treatment paradigms. Based on current available evidence, specialists at our institution and others currently advise a limited evaluation for specific symptoms as listed in the accompanying table (Table 1). PCPs should be equipped to evaluate alternative etiologies of symptoms, validate symptoms as potentially related to post-COVID when appropriate, and provide support as needed. Many patients will fully recover from their illness, albeit in a prolonged fashion. Clinicians should convey this to patients as encouragement and also be prepared to provide treatment and interventions that will support patients during their recovery. Table 1 outlines options for how PCPs may approach initial evaluation and management of a patient with postCOVID symptoms. As always, clinical care should start with a detailed history and physical and then judicious test ordering as needed. Table 1 is not meant to provide a list of necessary tests and interventions but rather is a comprehensive guide of options to consider or rule out if symptoms are particularly concerning or persist even after a period of "watchful waiting." In the sections that follow, we have provided additional context and explanation for the more unique or complex symptoms that a PCP may encounter in evaluating these patients.

\section{Respiratory Symptoms}

A persistent sensation of breathlessness (dyspnea) is a common finding despite normal examination findings and pulse oximetry after infection with COVID-19. The cause of this is likely multifactorial and may be impacted by thoughts, emotions, and behaviors that the person has associated or experienced with dyspnea. In a Norwegian prospective cohort study of patients surviving hospital admission for COVID-19, continued dyspnea did not necessarily correlate with lung function, computed tomography (CT) findings, or intensive care unit (ICU) admission. ${ }^{16}$

If a patient presents with continued dyspnea post-COVID-19 infection, the history and physical examination are important to assess whether this is a new symptom versus a continuation of prior symptoms. It is also important to discuss smoking/ vaping history, in addition to previously diagnosed medical conditions involving the pulmonary and cardiac systems. Paying attention to effort of breathing at rest, respiratory rate, heart rate, blood pressure, and pulse oximetry at rest and with exertion may be helpful. The 1-minute sit to stand test (Appendix) can help to quantify the effect of breathlessness on function and may be useful to track progress toward improvement over time.

Initial evaluation with a complete blood count (CBC) and basic metabolic panel (BMP) can help evaluate for other causes of dyspnea. Screening for obstructive sleep apnea (OSA) using the STOPBANG questionnaire as well as a chest radiograph (CXR) and a complete pulmonary function test (PFT) are also suggestions for evaluation. If there are concerning examination or PFT findings, a CT chest is recommended. Pulmonary consultation should be considered with abnormalities on imaging or PFT, abnormalities on examination such as dry/velcro crackles, or a history of progressively worsening dyspnea.

If the patient's evaluation yields no or only minimal abnormalities, reassurance and focusing on a cognitive-behavioral approach such as the breathing thinking functioning (BTF) model can be useful (see Figure 1 and symptom management approaches below). ${ }^{17}$ Breathing exercises (see "Breathing Techniques") and the use of an incentive spirometry may be helpful for recovery, especially in the presence of anxiety worsening the sensation of breathlessness. In addition, a relatively inexpensive home pulse-ox monitoring may help with patient reassurance.

\section{Breathing Techniques}

The following breathing techniques are examples of ways to breathe more efficiently, while enhancing relaxation and promoting awareness of the breath and its effects on the body.

The patient should be encouraged to practice 1 or more of the following breathing techniques 5 to 10 times per day and as needed during episodes of breathlessness to aid in relaxation and maximizing oxygenation:

1. Belly or diaphragmatic breathing is best practiced in a supine position. A patient should place one hand on the stomach and another on the chest. While inhaling 4 counts, an expansion of the stomach (not chest) is the goal. As the patient exhales on 4 counts the belly goes back down.

2. Pursed-lip breathing can be practiced in a relaxed sitting position. A patient should repeatedly breathe in through one's nose in 2 counts then purse lips to blow out slowly in 4 counts. 
Figure 1. An updated breathing thinking functioning model.

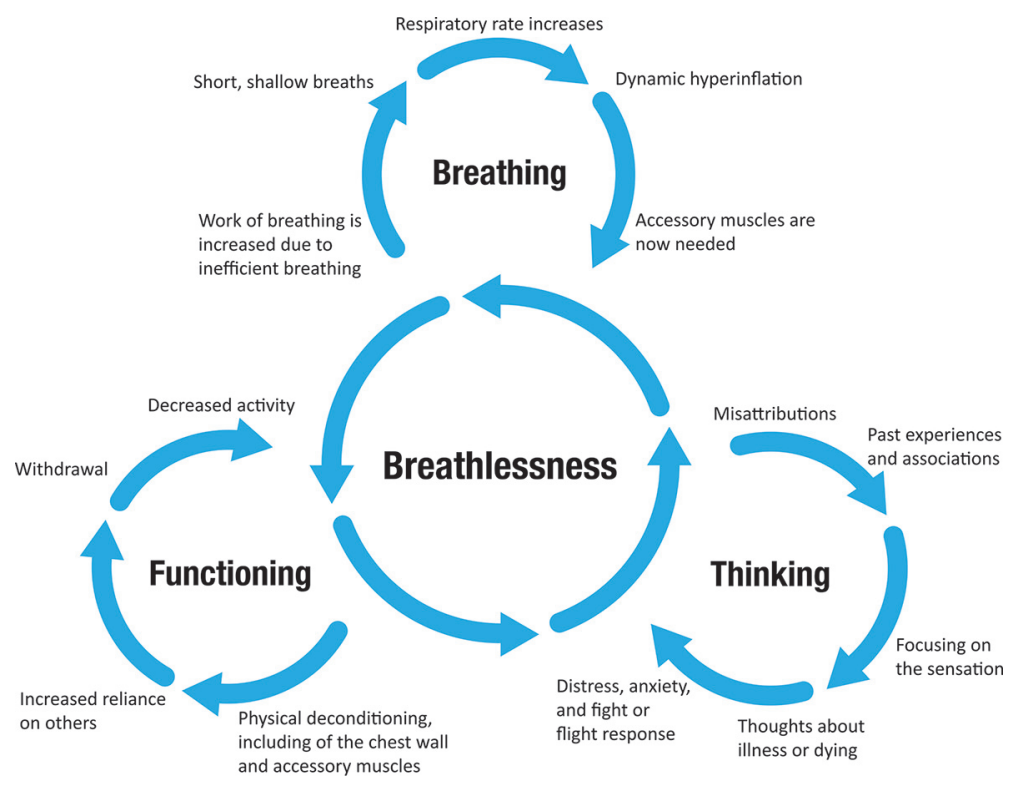

Table 2. Return to Exercise Guidance Chart

\begin{tabular}{|c|c|c|c|c|}
\hline \multirow[b]{2}{*}{ Recommendations } & \multicolumn{4}{|c|}{ Severity of Symptoms } \\
\hline & No Symptoms & Mild Symptoms & Moderate Symptoms* & $\begin{array}{c}\text { Severe Symptoms or } \\
\text { Hospitalized }\end{array}$ \\
\hline Rest & $\begin{array}{l}\text { No exercise for } 10 \text { days } \\
\text { from positive test result }\end{array}$ & $\begin{array}{l}\text { No exercise for } 10 \text { days or } \\
\text { more since symptom } \\
\text { onset AND no } \\
\text { symptoms for }>10 \text { days }\end{array}$ & $\begin{array}{l}\text { No exercise for } \\
>10 \text { days since } \\
\text { symptom onset AND } \\
\text { no symptoms for } \\
>10 \text { days }\end{array}$ & $\begin{array}{l}\text { See your medical } \\
\text { provider or } \\
\text { cardiologist before } \\
\text { returning to } \\
\text { exercise }\end{array}$ \\
\hline Cardiovascular testing & Not advised & Not advised & $\begin{array}{l}\text { Evaluation by a medical } \\
\text { provider and } \\
\text { consideration of } \\
\text { cardiovascular testing }\end{array}$ & \\
\hline $\begin{array}{l}\text { Return to exercise } \\
\text { guidance }\end{array}$ & $\begin{array}{l}\text { Paced and gradual return } \\
\text { to activity }\end{array}$ & $\begin{array}{l}\text { Paced and gradual return } \\
\text { to activity }\end{array}$ & $\begin{array}{l}\text { Clearance from medical } \\
\text { provider to return to } \\
\text { activity }\end{array}$ & \\
\hline
\end{tabular}

Note: Stop activity and see your medical provider if new symptoms such as dizziness, chest pain, shortness of breath, or palpitations develop while returning to exercise.

*Patients above 65 years old or those with significant cardiovascular risk factors such as diabetes, hypertension, coronary artery disease, atrial fibrillation, or kidney disease would also fall into this category.

3. Boxed breathing involves inhaling on 4 counts, holding on 4 counts, exhaling on 4 counts, and holding again on 4 counts.

\section{Cognitive Symptoms}

Patients can also report cognitive changes, such as "brain fog," decreased concentration, or memory difficulties. Preliminary data suggest post-COVID cognitive impairment is most common following a medically complex course (development of ICU delirium, multiorgan involvement, and so on). ${ }^{18,19}$
Initial workup may include $\mathrm{CBC}$, complete metabolic panel (CMP), vitamin B12, vitamin D-3, and thyroid stimulating hormone (TSH). The provider can offer much benefit by giving education on the mind-body connection, setting expectations for improvement while being mindful not to provide guarantees about the final outcome at this early stage in our understanding of the disease, and helping the patient to optimize other factors that may be impacting functional cognition, including mood, pain, and medication effects. While the outcomes 
Figure 2. Possible factors influencing symptom reporting acutely, postacutely, and long after a CoVID-19 infection. PTSD, post-traumatic stress disorder.

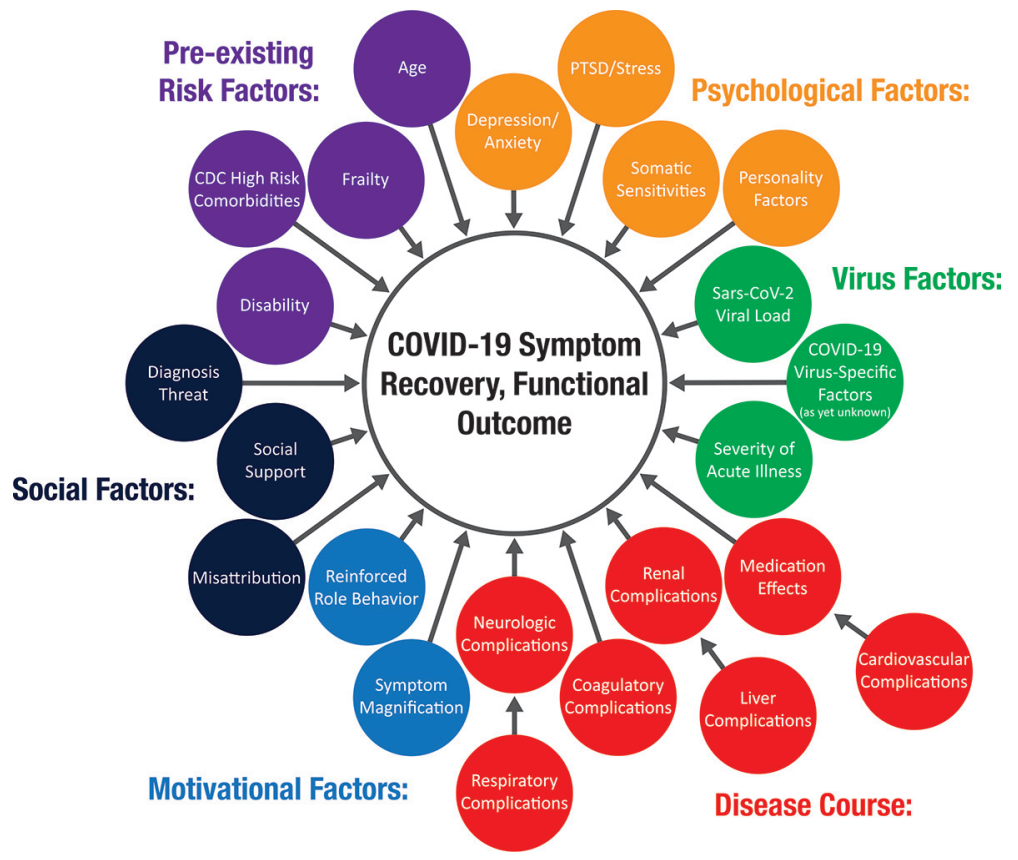

literature continues to evolve, these nondisease-specific factors are most likely to be a primary contributor to cognitive symptom experience in cases where patients had an uncomplicated medical course but are reporting significant cognitive changes (Figure 2). This is supported by findings of only mild cognitive change for most recovered patients, with those changes associated with degree of inflammation, when controlling for factors such as mental health disorders, need for psychotropic medications, and history of substance abuse. ${ }^{19}$ The threshold for referral for psychotherapy should be low, given the impact mood and distress can have on functional cognition.

If moderate to severe cognitive impairment is suspected in the context of a more severe, medically complex case of COVID-19, further testing may be indicated including: HIV, rapid plasma reagin (RPR), folate, thiamine, and neuropsychological testing. Magnetic resonance imaging (MRI) may be useful if patients have had moderate to severe COVID, are $>50$ years of age, or have focal neurologic findings. ${ }^{20,21}$ It will be important to interpret these often-nonspecific MRI findings in the context of known high rates of cerebrovascular disease, including silent disease, in the general public. $^{22}$ In these cases, it would be difficult if not impossible for the provider to determine if the MRI findings are specific to the COVID diagnosis, and misattribution to the virus may inadvertently discourage the patient from managing ongoing modifiable vascular risk factors with diet, exercise, and appropriate medications or medical devices such as continuous positive airway pressure (CPAP). Referral for formal neuropsychological evaluation should be considered for severe or persistent symptoms. Recommendations from this evaluation can help determine appropriateness of cognitive rehabilitation or other treatments, as well as decisions about return to work, school, or other activities of daily living. Treatment with neurostimulants is generally discouraged given the potential for addiction, sleep disruption, and increased anxiety but may be considered depending on level of functional disruption. Ideally, cognitive impairment will have been confirmed by a neuropsychologist before trialing neurostimulants.

\section{Neurological Symptoms}

Neurological symptoms, which have been documented in previous $\mathrm{CoV}$ endemics, ${ }^{23}$ may include chronic headaches, weakness, paresthesias, and dizziness. ${ }^{4}$ Patients will describe the headache as constant pressure, often waxing and waning. ${ }^{20}$ Other 
neurologic symptoms, including paresthesias and dizziness, can occur in $10 \%$ to $15 \%$ of COVID-19 survivors. ${ }^{23}$ Paresthesias can have a myriad of presentations including changing locations, focal, or diffuse. ${ }^{20}$

After a detailed history of the individual's current symptoms, pattern of symptoms, COVID history, and past medical history are obtained, a small and focused workup can be appropriate based on the symptoms. ${ }^{20}$ Neurologic imaging (MRI brain) for individuals with headache is recommended if there is an escalating pattern or additional red flags of a chronic headache (ie, "worst headache of life," associated neurologic findings) or if a patient has moderate to severe COVID-19, is older than 50, or has comorbidities. ${ }^{20,21}$ For many cases of chronic headaches, lifestyle modifications in exercise, diet, and sleep are recommended. For individuals presenting with other neurologic symptoms such as paresthesias or dizziness, initial workup should include CBC, CMP, $\mathrm{TSH}$, vitamin $\mathrm{D}$, vitamin $\mathrm{B} 12$, and hemoglobin A1c (if paresthesias are present). An MRI brain would be indicated if neurologic red flag symptoms (as discussed above) are present or if weakness, paresthesias, or impaired mobility are present. Reassurance is key for patients, especially in the presence of a normal workup and mild symptoms. ${ }^{23}$ There are medications that may alleviate this neurological pain, including gabapentin, pregabalin, tricyclics, or serotoninnorepinephrine reuptake inhibitors (SNRIs), and/or physical or occupational therapy can be suggested. A patient should be referred to a multidisciplinary postCOVID clinic if symptoms have not lessened since initial treatment, or neurology, psychology and/or physical medicine and rehabilitation $(\mathrm{PM} \& \mathrm{R})$ if clinic time is not available or applicable.

\section{Cbronic Fatigue}

Chronic fatigue is 1 of the most commonly reported symptoms in patients with a history of COVID-19, with $>50 \%$ incidence reported in multiple studies. ${ }^{2,6,24,25}$ Presence or severity of fatigue is not necessarily associated with severity of illness or with routine lab markers of inflammation. ${ }^{25}$ The underlying cause of fatigue in post-COVID syndrome is under investigation. A study by Ortelli et $\mathrm{al}^{26}$ suggests that COVID-19-associated inflammation may lead to GABA-ergic impairment, explaining apathy and executive functioning deficits. Another proposed framework suggests that fatigue is dependent on both conditional (task, environment, physical and mental capacity of individuals) and physiologic (central and psychological) factors. ${ }^{27}$ In the absence of evidence addressing specific treatments for COVID-related fatigue, clinicians can apply their normal approach for managing chronic fatigue due to other conditions. Graded or paced exercise of low impact and short duration may be recommended. Exercise should be undertaken cautiously and reduced if exacerbations in other symptoms such as breathlessness or myalgia occur. ${ }^{28}$ In severe cases, stimulants may be considered, but patients should be monitored carefully for adverse reactions, particularly if cardiovascular complications are present. In all cases, patients should be provided empathy and reassurance.

\section{Insomnia/Mood Changes}

Mood and sleep changes are fairly common after COVID-19 infection. Following severe infection requiring an ICU stay, preliminary studies show the psychological impact is similar to what is seen following prolonged ICU stays for similar or co-occurring diagnoses ${ }^{18}$ and may include depression, anxiety, and/or a post-traumatic response. Although we anticipate a more pronounced effect on mood and sleep with more severe cases, those with premorbid vulnerabilities may experience significant effects in these areas with even a mild case.

For mood symptoms, referral to psychotherapy should be considered as a first-line treatment, given adjustment difficulties may be the primary driver or a contributory factor for other symptoms (eg, anxiety may be driving sleep disruptions, palpitations or lightheadedness, depression may be driving slowed cognitive processing or fatigue, and so forth). PCPs should refer to therapists who use evidence-based therapies, which may include cognitive behavioral therapy (CBT), acceptance and commitment therapy (ACT), or trauma-processing protocols such as cognitive processing therapy (CPT) or prolonged exposure (PE). ${ }^{29}$

An initial workup for sleep disruption should include TSH, CBC, and an iron study. A PCP should also screen for OSA, using the STOPBANG questionnaire. First-line treatment should include cognitive behavioral therapy for insomnia $(\mathrm{CBT}-\mathrm{I})^{29}$ and sleep hygiene exercises, though sleep aids, including melatonin, mirtazapine, gabapentin, and amitriptyline (if paresthesias or headaches are also present) can also be considered. A patient's age and cognitive status should be considered when prescribing sleep aids, as some of these medications have a strong cognitive burden. Additional sleep 
medicine may be recommended if symptoms have not lessened with these treatments.

\section{Dysautonomia}

Dysautonomia or the feeling of lightheadedness or palpitations can occur in patients who have recovered from COVID-19. Given the overlap with other clinical manifestations of post-COVID symptoms, it is difficult to accurately report the full impact of dysautonomia. ${ }^{30}$ Initial workup using a limited, focused approach should be concentrated on identifying any underlying cause. Laboratory evaluation should include a CBC, CMP, and TSH. In-office evaluation for orthostatic hypotension should also be performed, and one can consider referral for tilt table testing. ${ }^{31}$

Treatment should be tailored to the symptoms and response but should focus on hydration, increasing salt intake, and use of compression stockings. ${ }^{20}$ The breathing techniques discussed above as well as meditation may be useful tools to alleviate some of these symptoms. If symptoms are severe or refractory, consider referral to cardiology or a multidisciplinary COVID team (if available) and possible use of midodrine, fludrocortisone, or $\beta$-blockers for hyperadrenergic POTS. ${ }^{31}$

\section{Anosmia and Smell Retraining}

In most cases, anosmia related to COVID-19 infection is described as sudden in onset, with short duration, and with quick recovery back to baseline taste or smell. While research suggests that most individuals find significant improvement in taste or smell within 7 to 10 days, it can last weeks or months. ${ }^{32}$ Although the explanation is currently not clear, ${ }^{33}$ possible explanations as to what may cause anosmia during COVID-19 infection include congestion, damage to the olfactory nerve, inflammation in cells within the lining of the nasal cavity, and viral effects on the cranial nerves within the brain. Underlying etiologies such as allergies should be considered. If allergic rhinitis or association congestion is present, nasal steroids may be useful for some patients and carry minimal risk. Smell retraining can be effective over time, while referral to an ear, nose, and throat (ENT) doctor should be considered if symptoms persist for longer than 6 months.

\section{Return to Exercise}

Patients are often eager to return to normal activities, including exercise, after recovering from
COVID-19. However, it is known that patients diagnosed with COVID are at risk of cardiac complications, including myocarditis, pericarditis, and arrhythmia. It would not be feasible to provide cardiac testing before all patients return to exercise. Kim et $\mathrm{al}^{34}$ have outlined a straightforward, targeted risk-stratification approach to advising patients based on their age, illness course, and level of athletic participation. In general, those who are asymptomatic or only mildly ill will need to abstain from exercise for 10 days and symptom resolution. For those with moderate to severe illness or who are hospitalized, they advise resting an additional 10 days beyond symptom resolution as well as testing with electrocardiogram (EKG), high-sensitivity troponin, and echocardiogram. If testing is normal, and for those who were asymptomatic or only mildly ill, they can proceed to gradual return to play. Kim et al further risk-stratify their guidance with specific return-toplay algorithms for athletes in competitive high school sports, recreational master athletes, and adult athletes in competitive sports. These algorithms serve as an excellent resource for clinicians.

We have adapted Table 2 to reflect published guidelines on return to exercise from Kim and colleagues $^{34}$ and Elliott et $\mathrm{al}^{35}$ to help guide clinicians and patients. Visit the link http://www.med.umich. edu/1libr/FamilyMedicine/PostCOVIDclinic/PostCOVID\%20ReturnToExercise.pdf for more detail and suggestions for patients.

\section{Discussion}

The management of acute COVID has been vigorously studied since the appearance of the virus in late 2019 and early 2020, but data on long-term effects of the disease are still emerging. In the absence of evidence-based resources for management of postCOVID symptoms, PCPs are relying on their existing knowledge base for treatment of their patients. As more data about management of this syndrome become available, rapid dissemination of this data to front-line PCPs is crucial. This table was our effort to make the currently available evidence accessible for our PCPs in a simple, easy-to-use format. Recognizing that both patients and providers would find it valuable, we also created educational resources that are readily available to address these various common postacute and chronic COVID symptoms.

Literature on post-COVID care is limited but rapidly developing as our experience with the novel 
virus expands. In addition, many health systems have developed different systems for managing post- and long-COVID patients that have not been published. As the knowledge base expands, recommendations on management will likely need to be altered. This work aligned with an institutional effort to create a multidisciplinary post-Covid clinic at MM. Not all hospital systems will have a multidisciplinary post-COVID clinic, and recommendations for referral will vary.

Future research could focus on validation of the proposed tool, as well as provider experience of the usefulness and reliability. It would also be beneficial to gather information, both evidence-based and patient-driven, on whether accepted approaches for management of common symptoms such as fatigue and headache are appropriate in these populations.

To see this article online, please go to: http://jabfm.org/content/ 34/6/1229.full.

\section{References}

1. Centers for Disease Control and Prevention. COVID data tracker. Available from: https://covid.cdc.gov/ covid-data-tracker/\#cases_casesper100klast7days Accessed June 15, 2021.

2. Garrigues E, Janvier P, Kherabi Y, et al. Postdischarge persistent symptoms and health-related quality of life after hospitalization for COVID-19. J Infect 2020;81:e4-e6.

3. Carfî A, Bernabei R, Landi F, for the Gemelli Against COVID-19 Post-Acute Care Study Group. Persistent symptoms in patients after acute COVID-19. JAMA 2020;324:603-5.

4. Petersen MS, Kristiansen MF, Hanusson KD, et al. Long COVID in the Faroe Islands-a longitudinal study among non-hospitalized patients. Clin Infect Dis 2020; ciaa1792.

5. Huang C, Huang L, Wang Y, et al. 6-month consequences of COVID-19 in patients discharged from hospital: a cohort study. Lancet 2021;397:220-32.

6. Huang Y, Pinto MD, Borelli JL, et al. COVID symptoms, symptom clusters, and predictors for becoming a long-hauler: looking for clarity in the haze of the pandemic. medRxiv 2021:2021.03.03.21252086.

7. Goërtz YMJ, Van Herck M, Delbressine JM, et al. Persistent symptoms 3 months after a SARS-CoV-2 infection: the post-COVID-19 syndrome? ERJ Open Res 2020;6.

8. Alwan NA, Johnson L. Defining long COVID: going back to the start. Med (N Y) 2021;2:501-4.

9. National Institute for Health and Care Excellence; 2020. Rapid guideline: managing the long-term effects of COVID-19. Available from: https:// www.nice.org.uk/guidance/conditions-and-diseases/ respiratory-conditions/covid19. Accessed March $15,2021$.

10. Carvalho-Schneider C, Laurent E, Lemaignen A, et al. Follow-up of adults with non-critical COVID-19 two months after symptom onset. Clin Microbiol Infect 2021;27:258-63.

11. Nabavi N. Long covid: how to define it and how to manage it. BMJ 2020;370:m3489.

12. Walsh-Messinger J, Manis H, Vrabec A, et al. The kids are not alright: a preliminary report of postCOVID syndrome in university students. medRxiv [Preprint] 2020; Nov 29:2020.11.24.20238261.

13. Office for National Statistics; 2020. Prevalence of long COVID symptoms and COVID-19 complications. Available from: https://www.ons.gov.uk/ peoplepopulationandcommunity/healthandsocialcare/ healthandlifeexpectancies/datasets/prevalenceoflongc ovidsymptomsandcovid19complications. Accessed March 15, 2021.

14. Royal College of General Practitioners; 2020. GPs will be at the forefront of helping patients cope with "lingering and difficult legacy: of COVID-19 - but will need support, says college. Available from: https:// www.rcgp.org.uk/about-us/news/2020/july/gps-willbe-at-the-forefront-of-helping-patients-cope-withlingering-and-difficult-legacy.aspx. Accessed March 29, 2021.

15. Ali MK, Shah DJ, Del Rio C. Preparing primary care for COVID-20. J Gen Intern Med 2020; 9:1-2.

16. Lerum TV, Aaløkken TM, Brønstad E, et al. Dyspnoea, lung function and CT findings 3 months after hospital admission for COVID-19. Eur Respir J 2021;57:2003448.

17. Spathis A, Booth S, Moffat C, et al. The breathing, thinking, functioning clinical model: a proposal to facilitate evidence-based breathlessness management in chronic respiratory disease. NPJ Prim Care Respir Med 2017;27:27.

18. Mcloughlin BC, Miles A, Webb TE, et al. Functional and cognitive outcomes after COVID19 delirium. Eur Geriatr Med 2020;11:857-62.

19. Zhou H, Lu S, Chen J, et al. The landscape of cognitive function in recovered COVID-19 patients. J Psychiatr Res 2020;129:98-102.

20. Navis A. 2021. Post-COVID neurological symptoms: experiences from our center [webinar]. Slides 33-52. Available from: https://emergency.cdc.gov/ coca/ppt/2021/012821_slide.pdf.

21. Clinician Outreach and Communication Activity (COCA) webinar; 2021. Treating long COVID: clinician experience with post-acute COVID-19 care. Available from: https://emergency.cdc.gov/coca/ calls/2021/callinfo_012821.asp.

22. Tong X, Yang Q, Ritchey MD, et al. The burden of cerebrovascular disease in the United States. Prev Chronic Dis 2019;16:E52. 
23. Barker-Davies RM, O'Sullivan O, Senaratne KPP, et al. The Sanford Hall consensus statement for post-COVID-19 rehabilitation. Br J Sports Med 2020;54:949-59.

24. Halpin SJ, McIvor C, Whyatt G, et al. Postdischarge symptoms and rehabilitation needs in survivors of COVID-19 infection: a cross-sectional evaluation. J Med Virol 2021;93:1013-22.

25. Townsend L, Dyer AH, Jones K, et al. Persistent fatigue following SARS-CoV-2 infection is common and independent of severity of initial infection. PLoS One 2020;15:e0240784.

26. Ortelli P, Ferrazzoli D, Sebastianelli L, et al. Neuropsychological and neurophysiological correlates of fatigue in post-acute patients with neurological manifestations of COVID-19: insights into a challenging symptom. J Neurol Sci 2021;420: 117271 .

27. Rudroff T, Fietsam AC, Deters JR, Bryant AD, Kamholz J. Post-COVID-19 fatigue: potential contributing factors. Brain Sci 2020;10:1012.

28. Greenhalgh T, Knight M, A'Court C, Buxton M, Husain L. Management of post-acute Covid-19 in primary care. BMJ 2020;370:m3026.

29. Society of Clinical Psychology (Division 12 APA); 2016. Resource page. Available from: https://div12. org/psychological-treatments/. Last accessed May, 15,2021 .

30. Raj SR, Arnold AC, Barboi A, Claydon VE, American Autonomic Society, et al Long-COVID postural tachycardia syndrome: an American Autonomic Society statement. Clin Auton Res 2021;31:365-4. Available from: https://link.springer.com/article/10.1007\% 2Fs10286-021-00798-2

31. Dani M, Dirksen A, Taraborrelli P, et al. Autonomic dysfunction in "long COVID": rationale, physiology and management strategies. Clin Med (Lond) 2021;21:e63-67.

32. Whitcroft KL, Hummel T. Olfactory dysfunction in COVID-19: diagnosis and management. JAMA 2020;323:2512-4.

33. Butowt R, von Bartheld CS. Anosmia in COVID19: underlying mechanisms and assessment of an olfactory route to brain infection. Neuroscientist 2020;1073858420956905.

34. Kim JH, Levine BD, Phelan D, et al. Coronavirus disease 2019 and the athletic heart: emerging perspectives on pathology, risks, and return to play. JAMA Cardiol 2021;6:219-27.

35. Elliott N, Martin R, Heron N, et al. Infographic: graduated return to play guidance following COVID19 infection. Br J Sports Med 2020;54:1174-5. 


\section{Appendix}

The 1-minute sit to stand test (1STST) was performed as previously described with a chair of standard height $(46 \mathrm{~cm})$ without armrests positioned against a wall. The patient was seated upright on the chair with knees and hips flexed at $90^{\circ}$, feet placed flat on the floor a hip-width apart, and arms held stationary by placing their hands on their hips. Patients were asked to perform repetitions of standing upright and then sitting down in the same position at a self-paced speed (safe and comfortable) as many times as possible for 1 minute. They were instructed not to use their arms for support while rising or sitting. Patients were permitted to rest during the 1 -minute period. The number of repetitions was recorded. The modified Borg scale (0 to 10 ) was used to assess dyspnea and fatigue immediately before and after each test. A finger pulse oximeter (Nellcor OxiMax N-65; Covidien, Minneapolis, $\mathrm{MN}$ ) was connected throughout the test for continuous recording of $\mathrm{SpO} 2$ and heart rate. A desaturation level $>4 \%$ was considered clinically significant for this study. 